stant, $T$ the absolute temperature, $n_{2}(r) / n$ the radial distribution function and $C_{k}, C_{p}, A_{k}, A_{p}, \gamma$ are numerical constants.

The first part of $\mu$ and $\lambda$ can be shown to reduce to the corresponding formulæ in gas theory ${ }^{2}$ for the case of rigid spheres, and the relation $\lambda=\mu C_{v}$, where $C_{v}=3 k / 2 m$, can be seen to hold for any model.

It should be possible to fix the constant coefficients if sufficient knowledge of the radial distribution function and its rate of change with density and temperature could be obtained. Theoretical investigations of this problem are now in progress in this Department.

I wish to express my thanks to Prof. Max Born, who suggested this problem to me, and to Dr. H. S. Green for his advice.

Department of Mathematical Physics,

$$
\text { L. M. YANG }
$$

University of Edinburgh. Dec. 18.

${ }^{3}$ Born, M., and Green, H. S., Proc. Roy. Soc., A, 188, 10 (1946). Green, H. S., Proc. Roy. Soc., A, 190, 455 (1947).

"Chapman, S., and Cowling, T. G., "The Mathematical Theory of Non-uniform Gases" (Camb. Univ. Press, 1939).

\section{A Bunsen Flame as a Column of Luminous Gas}

THE long duration of the luminosity (afterglow) of flame gases after the explosion of a well-mixed inflammable gaseous mixture contained in a large closed vessel, in which cooling takes place slowly', suggests that were it possible to secure steady stream. lined flow of the flame gases rising from a very large open flame, thus eliminating cooling due to turbulence, they would appear as a column of luminous gas many feet in height. Experimenting recently, we have been a little surprised to find how far we may go in this direction with a small Bunsen flame resulting from the combustion of fully aerated coal-gas. It is easily possible to obtain a column of luminous gas which, viewed in a dark room, extends to a height of $2 \mathrm{ft}$., whereas with the ordinary Bunsen flame luminosity ceases at a height of only 7 or 8 in.

All that is necessary is to hold an open-ended tube around the flame, as shown in Fig. a. It is important

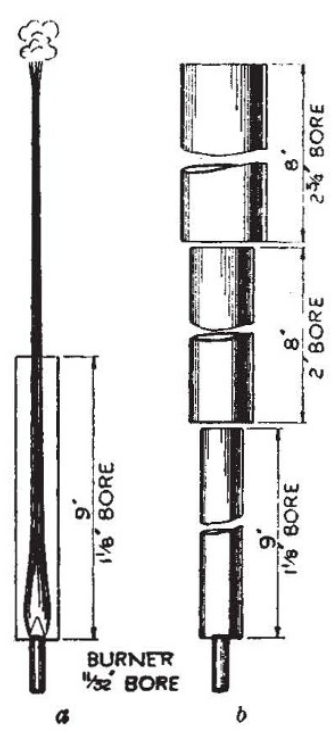
that a tube of the right diameter and length in relation to the burner diameter should be selected. The dimensions of the tube used in our experiments are given in the figure.

An even greater height of luminous gas columnapproaching $3 \mathrm{ft}$.- - may be attained by the use of a series of open-ended tubes of increasing diameter placed one above the other, as shown in Fig. $b$.

It would appear that the flame gases become embedded in a column of expanding air rising through the tube, and the turbulent mixing with cold air, which always takes place near the tip of an ordinary flame (see Schlieren photographs ${ }^{2}$ ), is thus inhibited. The provision of additional tubes above the first appears to assist in maintaining the streamline flow.

The first six inches or so of the flame gases are yellow in colour. They then turn to red and quickly afterwards to blue, which persists through the whole length of the column. The yellow coloration indicates continued combustion above the inner cone due to stratification of the gas mixture in the flame resulting from imperfect mixing of the coal-gas and air during their passage up the burner tube. When a perfectly mixed coal-gas and air mixture (with excess air) contained in a tank is supplied to the burner (with the air port closed) there is no yellow coloration-the flame gases attain their reddish colour immediately above the inner cone and this turns quickly to the characteristic blue afterglow which persists as before.

We have obtained a similarly extended afterglow when burning pure carbon monoxide in the Bunsen burner.

We would again like to suggest that it is difficult to account for the long duration of the afterglow unless the presence in the flame gases of metastable molecules formed during combustion is assumed ${ }^{1}$. The excited molecules responsible for the light emission may result from the collisions of these molecules.

A. S. LEAH

Engineering Department,

W. T. DAVID

University, Leeds.

Dec. 8.

${ }^{2}$ Nature, 159, 407 (1947).

${ }^{2}$ Gawthrop, Rev. Sci. Instr., 2, 522 (1931).

\section{Equations of Piezo-electricity}

Prof. W. G. CaDy's exposition of the basic equations of piezo-electricity in his recent book "Piezoelectricity" contains errors which should be brought to the attention of students and workers in this field.

On p. 44, the first thermodynamic potential is introduced in equation (1), but is inoorrectly expressed as a quadratic form in terms of elastic strains and electric field intensities within the piezo-electric material (we are considering only isothermal effects here). This potential is also called Green's strain energy function, and is identical with the Lagrange function for a statical conservative system. It is well known that, for a linear conservative system, this function can be written either as a quadratic form in the generalized co-ordinates, in which case it is sometimes called the 'first thermodynamic potential', or as a quadratic form in the generalized forces, when it is called the 'second thermodynamic potential'. For an electromechanical system, suitable generalized co-ordinates are elastic strain and electric displacement, and the corresponding generalized forces are elastic stress and electric field intensity. By using elastic strain and electric field intensity in equation (1), however, Prof. Cady mixes generalized co-ordinates and forces in the Lagrange function, which results in erroneous piezo-electric equations. The second thermodynamic potential is introduced in equation (2) on p. 44, and is correctly written as a quadratic form in elastic stress and electric field intensity. Equations based on (1) will be physically inconsistent with equations based on (2).

Theoretical conclusions drawn from the incorrect equation (1) by the usual Lagrange formalism 